\title{
Edentulism in Saudi Arabia and associated factors
}

\author{
Anas Omar Haroub ${ }^{1 *}$, Abdullah Abdu Khormi², Rawan Ahmad Yankesar ${ }^{3}$, \\ Shaden Abdullah Alhumaid ${ }^{4}$, Mohammad Ibrahim Aleissa ${ }^{5}$, Lubna Saleh Alotheem ${ }^{4}$, \\ Hussain Nayef Alsharif ${ }^{6}$, Khalid Abdulaziz Alghamdi ${ }^{7}$, Hassan Hamoud Alateeq ${ }^{8}$, \\ Ali Saeed Alshehri ${ }^{7}$, Alaa Mohammad Alaidarous ${ }^{9}$
}

\author{
${ }^{1}$ Department of Prosthodontics, Al Sundos Dental Care, Jeddah, Saudi Arabia \\ ${ }^{2}$ General Dentist, Ministry of Health, Jazan, Saudi Arabia \\ ${ }^{3}$ General Dentist, Ministry of Health, Al-Baha, Saudi Arabia \\ ${ }^{4}$ General Dentist, Ministry of Health, Alqassim, Saudi Arabia \\ ${ }^{5}$ Department of Dental Services, First Health Cluster, Riyadh, Saudi Arabia \\ ${ }^{6}$ General Dentist, Ministry of Health, Al-Jouf, Saudi Arabia \\ ${ }^{7}$ College of Dentistry, King Khalid University, Abha, Saudi Arabia \\ ${ }^{8}$ General Dentist, Ministry of Health, Hail, Saudi Arabia \\ ${ }^{9}$ College of Dentistry, Princess Nourah Bint Abdul Rahman University, Riyadh, Saudi Arabia
}

\author{
Received: 01 October 2021 \\ Accepted: 16 October 2021

\section{*Correspondence:} \\ Dr. Anas Omar Haroub, \\ E-mail: anaswars30@hotmail.com
}

Copyright: ( ) the author(s), publisher and licensee Medip Academy. This is an open-access article distributed under the terms of the Creative Commons Attribution Non-Commercial License, which permits unrestricted non-commercial use, distribution, and reproduction in any medium, provided the original work is properly cited.

\begin{abstract}
The epidemiology of edentulism is different across the different communities and the prevalence rates are also significantly different and it can be affected by various factors. There have been many investigations that reported the prevalence rates of edentulism across the different countries globally. However, not many investigations were published in Saudi Arabia and the published ones also report different findings. In the present study, we have reviewed the current studies in the literature to investigate the prevalence of edentulism and associated factors in Saudi Arabia. Our results indicated that the reported rates were conflicting among the different reports across the Kingdom and some of these rates are lower than other rates that were reported in some countries but higher than others also. Therefore, further nationwide research was still needed to formulate better evidence and help draw adequate interventions for the most vulnerable groups. Age, gender, educational levels, regional residency, socioeconomic status, depression and diabetes have all been reported to be correlated with edentulism. The prevalence of the condition has been reported to be highest among older age groups, while evidence is contradicting about the difference between both genders. Therefore, targeting vulnerable populations with early interventions can reduce the incidence rates and enhance the quality of life among these populations.
\end{abstract}

Keywords: Edentulsim, Demographics, Prevalence, Risk factors, Epidemiology, Teeth loss

\section{INTRODUCTIONS}

Serious worldwide concerns have been associated with the various oral diseases due to the significant burdens over the different healthcare systems, in addition to having a significant impact on the affected patients' lives. ${ }^{1}$ Tooth loss is a common oral condition and evidence indicates that periodontitis and dental caries are the most common causes of losing teeth. ${ }^{1,2}$ Disability secondary to oral diseases has also to be commonly associated with teeth loss and functional dentition has been demonstrated to be indicated when there are $\geq 20$ naturally present teeth., ${ }^{3,4}$ Furthermore, it has been 
demonstrated that the quality of life of the affected patients might be significantly impacted by teeth loss. ${ }^{5}$ Edentulism can significantly impact the nutritional status of the affected patients due to the reduced mastication abilities, in addition to decreasing the pleasure of eating. ${ }^{4,6}$

Evidence indicates that certain types of food are ingested is usually administered by patients with teeth loss.3,4 Furtherly, estimates show that even after managing these conditions with removable dentures, chewing abilities are still reduced in these patients as compared to the natural functions of the proper dentitions. The presence of chronic systemic conditions has also been correlated with the presence and development of edentulism. Besides, evidence also initiated an association between mortality and the development of disabilities and the presence of edentulism in the elderly. ${ }^{4,7}$ Accordingly, it has been concluded that the condition can affect the different aspects of the life of the affected patients including the physical, psychological and social parameters. Therefore, applying adequate interventions and management modalities should be established to achieve better carerelated outcomes.

The epidemiology of edentulism is different across the different communities, and the prevalence rates are also significantly different and can be affected by various factors. There have been many investigations that reported the prevalence rates of edentulism across the different countries globally. However, not many investigations were published in Saudi Arabia, and the published ones also report different findings. Therefore, we aimed to conduct the current investigation to study the prevalence and associated factors of edentulism in Saudi Arabia based on evidence from the relevant studies in the literature.

\section{METHODS}

This literature review was based on an extensive literature search in Medline, Cochrane, and EMBASE databases which was performed on 15th September 2021 using the medical subject headings (MeSH) or a combination of all possible related terms, according to the database. To avoid missing poetential studies, a further manual search for papers was done through Google Scholar while the reference lists of the initially included papers. Papers discussing the prevalence and associated factors of edentulism in Saudi Arabia were screened for useful information. No limitations were posed on date, language, age of participants or publication type.

\section{DISCUSSION}

Evidence in the current literature indicated that edentulism was a serious issue that should be managed to enhance the different aspects of the quality of life of the affected patients. In Saudi Arabia, we have identified a number of investigations that reported the prevalence and other epidemiological parameters of edentulism across the Kingdom. However, it should be noted that the evidence was based on a minimal number of investigations and further studies were encouraged to strengthen the current evidence and enhance the quality of reporting. In a previous cross-sectional investigation by Fouda et al conducted in Dammam city, the reported prevalence rate of partial edentulism was $74 \%$ of the included population. ${ }^{8}$ Besides, the authors also reported that $8 \%$ of their population had a single jaw edentulousness and only $6 \%$ had complete edentulism. The reported rate in this investigation was significantly lower than the reported prevalence rates among others that were conducted in Saudi Arabia and outside it. ${ }^{9-11}$ For instance, previous investigations in Saudi dental clinics and university settings and other countries showed that the prevalence of edentulism was estimated to be 13$17 \%{ }^{9,12}$ On the other hand, the reported prevalence rate was significantly lower than this reported in another investigation that was conducted in Riyadh city across different dental care settings by Almusallam et al which reported a rate of $1.8 \%$ only. ${ }^{13}$ Besides, it had been reported that $69 \%$ of their included population had at least one missing tooth and among these patients, $2.6 \%$ had complete edentulism. In Aljouf university, a crosssectional investigation was also conducted by Fayad et al14 and reported a prevalence rate of class III partial edentulism was $64.1 \%$ and $67.2 \%$ in the mandibular and maxillary arches, respectively. ${ }^{14}$ The prevalence of class II, on the other hand, was significantly lower in the mandibular and maxillary arches, being $14.8 \%$ and $16.3 \%$, respectively. In a nationwide investigation by AlZahrani et al the estimated prevalence rate was $4.5 \%$ of edentulism and the lack of functional dentition was prevalent in $10.3 \%$ of the included population. ${ }^{15}$ In AlQassim region, Saudi Arabia, Almutairy et al reported that the prevalence rate of edentulism was $62.5 \% .{ }^{16}$ It was also reported by the investigation by Heidari et al that most of the included participants in their investigation (94.4\%) were observed to have $\geq 1$ missing tooth. ${ }^{17}$

Studies from Europe and North America also indicated that the prevalence of edentulism had significantly increased over the past years. ${ }^{5,10,18}$ A previous investigation in Mexico that included individuals $\geq 18$ years old concluded that the prevalence of edentulism was estimated to be $6.3 \% .{ }^{19}$ Another investigation that included participants from the United Kingdom also reported that the prevalence of edentulism was $5 \%$ and $15 \%$ among individuals that were 55-64 and 65-74 years old, respectively. ${ }^{20}$ This indicated the impact of age on having edentulism among this population, which was also indicated among the different investigations in the Kingdom and was discussed later in this article. In 20092012, estimates from the United States indicated that the prevalence of edentulism was found to be $4.9 \%$ among $\geq 15$ year old participants. ${ }^{18}$ In the United States, Kim et al also conducted a similar estimate and reported that a total of $25.6 \%$ of their included population had $\geq 1$ missing tooth. ${ }^{21}$ High rates were also noticed in the previous 
investigation by Slade et al which was noticed to be higher than the estimates that were reported among some of the included investigations. ${ }^{22}$ On the other hand, several investigations from the African continent indicated that the prevalence of edentulism was significantly lower than these reported rates in the Kingdom of Saudi Arabia and other worldwide countries. $^{23-25}$ Investigations from Ghana and Nigeria have concluded that the prevalence of edentulism was found to be $2.8 \%$ and $1.3 \%$ among $\geq 50$ and $\geq 65$ year old participants, respectively. ${ }^{24,25}$

It should be noted that the number of investigations that were conducted in Saudi Arabia and the different Arabic countries was still minimum and required more researchers to conduct further investigations to additionally validated the evidence of the current investigations. ${ }^{12,23,26}$ The difference in prevalence rates among the different studies from different regions across the Kingdom and worldwide might be attributable to the different ages of the included population and the different exposure to the different demographics and risk factors that might increase the risk and prevalence of edentulism. Another essential factor to be considered and has been previously reported was the geographical differences among the different studies as some investigations included only patients from urban and/or rural areas, unlike others which might include participants from a single community or both of the communities, which might have different habitual and cultural differences. ${ }^{13,22}$ This had been furtherly indicated by the results of the previous investigation by Khazaei et al that concluded that the prevalence rate among developed countries seemed to be steadily decreasing, unlike developing countries where estimates indicated that the prevalence rates were increasing. ${ }^{27}$ On the other hand, another investigation by Douglass et al demonstrated that the increasing prevalence rates among the different communities were attributable to the increasing trends in population aging and related comorbidities that might significantly impact oral health. ${ }^{28}$

Many factors in the literature have been reported in association with edentulism. Among these factors, age had been reported as a significant contributor to the development of edentulism among the various studies in the literature, both globally and at a national level across the Kingdom. ${ }^{10,12,29}$ This association can be attributed to the fact that elderly patients usually suffered from systemic disorders and reduced general health status, which might limit their activities and taking adequate care of their oral health. ${ }^{30}$ The previous investigation by Fouda et al reported that age and male gender were significantly correlated with edentulousness and the number of missing teeth, respectively. ${ }^{8}$ They indicated that the prevalence of the condition was found the highest among participants $\geq 70$ years old. It had been furtherly demonstrated that partial and complete edentulism and single jaw edentulism were significantly associated with the presence of diabetes as associated morbidity. The previous multi-center investigation that was conducted in Riyadh city by Almusallam et al also indicated that age was significantly correlated with the development of complete edentulism. ${ }^{13}$ The authors also reported that $82 \%$ of these patients were $\geq 55$ years old, indicating a significant correlation between age and complete edentulism. The authors also reported that educational level was significantly correlated with edentulism. On the other hand, neither gender nor geographical region was significantly correlated. Behavioral variables were also reported to have a significant impact on the development of edentulism as the authors of this investigation reported that smoking habits, last dental visit and reason for the last visit were also significantly correlated with the development of edentulism in this Saudi population. It had also been demonstrated that edentulism increased with age in the cross-sectional investigation by Fayad et al as the authors reported that patients within the age group 41-50 years old had the highest prevalence rates of class I and II, while class III was highly prevalent among the $31-40$ years old age group. ${ }^{14}$ Aging was a significant healthcare issue to the different global healthcare systems because many diseases were usually associated with aging, in addition to the increased morbidity and healthcare-related burdens to the affected patients. Therefore, efforts should be adequately applied to enhance the healthcare outcomes to these groups, probably by applying adequate early interventions and increasing awareness and quality of care to the younger population to decrease the incidence of the different disease conditions. In a previous investigation in Canada, the authors reported that due to the increasing trend in the quality of dental care, the prevalence of edentulism has significantly decreased. However, they also reported that concerns regarding this expected reduction were also raised due to the increasing trends in the aging population. ${ }^{31}$ Accordingly, these findings indicated the significance of the different demographics as age and gender on the prevalence of edentulism across the different worldwide communities.

Depression had also been reported as a risk factor for developing edentulism in the nationwide investigation by Al-Zahrani et al which estimated that the prevalence of the condition was significantly higher in the group with depression rather than the other group that did not have it. ${ }^{15}$ The authors of this investigation also indicated that smoking, age, gender, educational level, presence of diabetes, alcohol consumption, body mass index, race and socioeconomic status were all significantly correlated with developing edentulism. Male gender seemed to be more correlated with a higher prevalence of edentulism. These results have been reported in Saudi Arabia and other worldwide investigations..$^{9,12,26,32}$ In Brazil, it was also reported that being male was associated with an increased risk of losing teeth. ${ }^{29}$ On the other hand, other conflicting findings were also reported by other investigations from different countries, where being male was associated with a higher risk and a higher prevalence than males. ${ }^{10,24,33}$ 


\section{CONCLUSION}

Nationwide research is still needed to formulate better evidence and help draw adequate interventions for the most vulnerable groups. Age, gender, educational levels, regional residency, socioeconomic status, depression and diabetes have all been reported to be correlated with edentulism. The prevalence of the condition has been reported to be highest among older age groups, while evidence is contradicting about the difference between both genders. Therefore, targeting vulnerable populations with early interventions can reduce the incidence rates and enhance the quality of life among these populations.

Funding: No funding sources Conflict of interest: None declared

Ethical approval: Not required

\section{REFERENCES}

1. Peres MA, Macpherson LMD, Weyant RJ, Daly B, Venturelli R, Mathur MR, et al. Oral diseases: a global public health challenge. Lancet. 2019;394(10194):249-60.

2. Rozier RG, White BA, Slade GD. Trends in oral diseases in the U.S. population. J Dent Educ. 2017;81(8):97-109.

3. Parker ML, Thornton-Evans G, Wei L, Griffin SO. prevalence of and changes in tooth loss among adults aged $\geq 50$ years with selected chronic conditions-United States, 1999-2004 and 20112016. MMWR. 2020;69(21):641-6.

4. Griffin SO, Jones JA, Brunson D, Griffin PM, Bailey WD. Burden of oral disease among older adults and implications for public health priorities. Am J Pub Health. 2012;102(3):411-8.

5. Polzer I, Schimmel M, Müller F, Biffar R. Edentulism as part of the general health problems of elderly adults. Int Dent J. 2010;60(3):143-55.

6. Bortoluzzi MC, Traebert J, Lasta R, DaRosa TN, Capella DL, Presta AA. Tooth loss, chewing ability and quality of life. Contemp Clinic Dentist. 2012;3(4):393-7.

7. Holm-Pedersen P, Schultz-Larsen K, Christiansen $\mathrm{N}$, Avlund K. Tooth loss and subsequent disability and mortality in old age. J Am Geriatr Soc. 2008;56(3):429-435.

8. Fouda SM, Al-Harbi FA, Khan SQ, Virtanen JI, Raustia A. Missing teeth and prosthetic treatment in patients treated at college of dentistry, university of Dammam. Int J Dentist. 2017;2017:7593540.

9. Hoover J, McDermott R. Edentulousness in patients attending a university dental clinic. J Canadian Dent Assoc. 1989;55(2):139-40.

10. Haikola B, Oikarinen K, Söderholm AL, RemesLyly T, Sipilä K. Prevalence of edentulousness and related factors among elderly Finns. J Oral Rehabilit. 2008;35(11):827-35.

11. Nazliel HE, Hersek N, Ozbek M, Karaagaoglu E. Oral health status in a group of the elderly population residing at home. Gerodontology. 2012;29(2):761-7.

12. Al-Ghannam N, Khan N, Al-Shammery A, Wyne A. Trends in dental caries and missing teeth in adult patients in Al-Ahsa, Saudi Arabia. Saudi Dent J. 2005;17(2):57-62.

13. Almusallam SM, AlRafee MA. The prevalence of partial edentulism and complete edentulism among adults and above population of Riyadh city in Saudi Arabia. J Fam Med Prim Care. 2020;9(4):1868-72.

14. Fayad MI, Baig MN, Alrawaili AM. Prevalence and pattern of partial edentulism among dental patients attending college of dentistry, Aljouf university, Saudi Arabia. J Int Soc Prevent Commun Dent. 2016;6(3):187-91.

15. Al-Zahrani MS, Alhassani AA, Melis M, Zawawi $\mathrm{KH}$. Depression is related to edentulism and lack of functional dentition: an analysis of NHANES data, 2005-2016. J Pub Health Dentist. 2020.

16. Almutairy A, Mohan M. Prevalence of partial edentulism among young Saudi women of Qassim and their perception of early tooth loss. Int $\mathbf{J}$ Dent Res. 2017;5:172-6.

17. Heidari E, Banerjee A, Newton JT. Oral health status of non-phobic and dentally phobic individuals; a secondary analysis of the 2009 adult dental health survey. Br Dent J. 2015;219(9):9.

18. Slade G, Akinkugbe A, Sanders A. Projections of US edentulism prevalence following 5 decades of decline. J Dent Res. 2014;93(10):959-65.

19. Medina-Solís CE, Pérez-Núñez R, Maupomé G, Avila-Burgos L, Pontigo-Loyola AP, Patiño-Marín $\mathrm{N}$, et al. National survey on edentulism and its geographic distribution, among Mexicans 18 years of age and older (with emphasis in WHO age groups). J Oral Rehabilit. 2008;35(4):237-44.

20. Steele J, Treasure E, O'sullivan I, Morris J, Murray J. Adult dental health survey 2009: transformations in British oral health 1968-2009. Br Dent J. 2012;213(10):523-7.

21. Kim S, Park S, Lin M. Permanent tooth loss and sugar-sweetened beverage intake in U.S. young adults. J Pub Health Dent. 2017;77(2):148-54.

22. Slade GD, Akinkugbe AA, Sanders AE. Projections of U.S. Edentulism prevalence following 5 decades of decline. J Dent Res. 2014;93(10):959-65.

23. Khalifa N, Allen PF, Abu-bakr NH, Abdel-Rahman ME. Factors associated with tooth loss and prosthodontic status among Sudanese adults. J Oral Sci. 2012;54(4):303-12.

24. Hewlett SA, Calys-Tagoe BN, Yawson AE. Prevalence and geographic distribution of edentulism among older $\mathrm{G}$ hanaians. J Pub Health Dentist. 2015;75(1):74-83.

25. Taiwo JO, Omokhodion F. Pattern of tooth loss in an elderly population from Ibadan, Nigeria. Gerodontology. 2006;23(2):117-22.

26. Al-Dwairi ZN. Complete edentulism and socioeconomic factors in a Jordanian population. Int J Prosthodont. 2010;23(6):541. 
27. Khazaei S, Firouzei MS, Sadeghpour S, Jahangiri P, Savabi O, Keshteli AH, et al. Edentulism and tooth loss in Iran: SEPAHAN systematic review no. 6. Int J Prevent Med. 2012;3(1):42-7.

28. Douglass CW, Shih A, Ostry L. Will there be a need for complete dentures in the United States in 2020 ? J Prosthet Dentist. 2002;87(1):5-8.

29. DeMarchi RJ, Hilgert JB, Hugo FN, Santos CMd, Martins AB, Padilha DM. Four-year incidence and predictors of tooth loss among older adults in a southern B razilian city. Commun Dentist Oral Epidemiol. 2012;40(5):396-405.

30. Avlund K, Holm-Pedersen P, Schroll M. Functional ability and oral health among older people: a longitudinal study from age 75 to 80 . J Am Geriatr Soc. $2001 ; 49(7): 954-62$.

31. Thompson GW, Kreisel PS. The impact of the demographics of aging and the edentulous condition on dental care services. J Prosthet Dent. 1998;79(1):56-9.

32. Eustaquio-Raga MV, Almerich-Silla JM. Factors associated with edentulousness in an elderly population in Valencia (Spain). Gaceta Sanitaria. 2013;27:123-7.

33. Russell SL, Gordon S, Lukacs JR, Kaste LM. Sex/Gender differences in tooth loss and edentulism: historical perspectives, biological factors, and sociologic reasons. Dent Clinic. 2013;57(2):317-37.

Cite this article as: Haroub AO, Khormi AA, Yankesar RA, Alhumaid SA, Aleissa MI, Alotheem LS, et al. Edentulism in Saudi Arabia and associated factors. Int J Community Med Public Health 2021;8:5614-8. 